\title{
Is avoidant disorder part of the social phobia spectrum in a referred sample of Brazilian children and adolescents?
}

D. Denardin, T.L. Silva, T.G. Pianca and L.A. Rohde

\author{
Serviço de Psiquiatria da Infância e Adolescência, \\ Hospital de Clínicas de Porto Alegre, Universidade Federal do Rio \\ Grande do Sul, Porto Alegre, RS, Brasil
}

\author{
Correspondence \\ L.A. Rohde \\ Serviço de Psiquiatria da Infância \\ e Adolescência \\ HCPA, UFRGS \\ Rua Ramiro Barcelos, 2350 \\ 90035-003 Porto Alegre, RS \\ Brasil \\ Fax: +55-51-3316-8294 \\ E-mail: Irohde@terra.com.br \\ Research partially supported by \\ FIPE-HCPA. D. Denardin and \\ T.L. Silva received support from \\ FAPERGS, and T.G. Pianca received \\ support from $\mathrm{CNPq} / \mathrm{PIBIC}$.
}

Received May 27, 2003 Accepted February 18, 2004

\begin{abstract}
The diagnosis of avoidant disorder was deleted from the Diagnostic and Statistical Manual of Mental disorders - fourth edition (DSM-IV) based on a 'committee decision' suggesting that avoidant disorder is part of the social phobia spectrum. The objective of the present study was to examine the nature of this clinical association in a referred sample of Brazilian children and adolescents. We assessed a referred sample of 375 youths using semi-structured diagnostic interview methodology. Demographic (age at admission to the study and sex) and clinical (level of impairment, age at onset of symptoms and pattern of comorbidity) data were assessed in subsamples of children with avoidant disorder $(\mathrm{N}=7)$, social phobia $(\mathrm{N}=26)$, and comorbidity between both disorders $(\mathrm{N}=24)$. Although a significant difference in the male/female ratio was detected among groups $(\mathrm{P}=0.03)$, none of the other clinical variables differed significantly among subjects that presented each condition separately or in combination. Most of the children with avoidant disorder fulfilled criteria for social phobia. Thus, our findings support the validity of the conceptualization of avoidant disorder as part of the social phobia spectrum in a clinical sample.
\end{abstract}

Key words

- Anxiety disorders

- Avoidant disorder

- Social phobia

- Mental disorder diagnosis
Although anxiety disorder is one of the most prevalent mental disorders in children and adolescents, very few studies have been conducted to assess and clarify its symptomatology, frontiers and clinical validity in youths (1). Anxiety disorders in children include a variety of entities such as conditions with diagnostic criteria derived from adult samples (panic disorder, agoraphobia, simple phobia, social phobia (SP), obsessive-compulsive disorder, post-traumatic stress disorder, generalized anxiety disorder), and those first recognized in childhood (separation anxiety disorder, overanxious disorder, and avoidant disorder, AD) (2).

SP is characterized by an excessive fear of observation or scrutiny in discrete performance situations, such as public speaking, writing or eating in front of other people (3). In clinical samples, the diagnosis of SP is more likely to be made in boys (4). AD is characterized by marked avoidance of contact with unfamiliar persons causing impairment in social relationships associated with a desire for contact with family members. The disorder should begin after 2.5 years of age 
in order to be differentiated from normal stranger anxiety that may occur up to this age (4).

The fourth edition of the Diagnostic and Statistical Manual of Mental Disorders (DSM-IV) (5) introduced several modifications in the section on disorders usually first diagnosed during infancy, childhood, or adolescence. The diagnosis of $\mathrm{AD}$ presented in the previous versions of the Manual (DSMIII and DSM-III-R) $(6,7)$ was deleted from DSM-IV. The rationale behind this decision was that $\mathrm{AD}$ is nothing more than a precocious variant of SP in children and adolescents. Thus, children with the symptoms of avoidance of contact with unfamiliar persons should receive a diagnosis of SP (3). The only study found in the literature that assessed several clinical markers (demographic variables and pattern of comorbidities) in three different groups (AD, SP, and $\mathrm{AD}+\mathrm{SP}$ ) did not demonstrate significant differences among groups, supporting the notion that AD overlaps with SP (8). Further indirect evidence of overlapping between the two disorders comes from a study of 100 adults with SP. At least $25 \%$ of the patients had a childhood history of AD (9). However, it is important to note that the decision to abolish the diagnosis of AD from the DSMIV was a 'committee decision' not based on strong scientific evidence, since no other investigations of the subject were found in the literature.

Due to the limited number of studies on the relationship of $\mathrm{AD}$ to $\mathrm{SP}$, we conducted a study to examine the nature of this clinical association in a referred sample of Brazilian children and adolescents. Based on the literature, we expected to find no significant clinical differences in demographic variables or pattern of comorbidities between a sample of children that fulfilled the diagnosis of $\mathrm{AD}$ and two other independent samples of children, one consisting of SP patients and the other of children diagnosed with both conditions. If this were the case, the justification for eliminating the diagnosis of $\mathrm{AD}$ from the DSM-IV would be stronger.

The sample comprised all children and adolescents evaluated at our Psychopharmacology outpatient unit in the Division of Child Psychiatry, Hospital de Clínicas de Porto Alegre (HCPA) from 1997 to the middle of 2002. We also included data for children and adolescents collected in our ADHD outpatient program and data for patients evaluated in private offices by 5 child psychiatrists (members of our team) during the same period. Subjects with AD and/or SP who fulfilled the criteria for the diagnosis of Pervasive Development Disorder were excluded from the study.

The diagnosis of mental disorders was made in a three-stage process: a) evaluation with a semi-structured interview (Schedule for Affective Disorders and Schizophrenia for School-Age Children, Epidemiological Version - K-SADS-E) (10) applied to the parents by trained assistants. Inter-rater reliability for derived diagnoses was evaluated previously in a study using videotapes of the interviews conducted by the research assistants when applying the K-SADS-E (11). The kappa $(\kappa)$ coefficients for mental disorders ranged from 0.79 to $0.96(\mathrm{P}<0.001)$. $\mathrm{b})$ Review of each diagnosis reached through the K-SADS-E by a clinical committee chaired by an experienced child psychiatrist (L.A.R.). c) Clinical evaluation of mental disorders using DSM-IV criteria by a child psychiatrist who previously had received the results of the K-SADS-E and conducted interviews with parents (usually the mother) and the child or adolescent. When a diagnostic disagreement occurred in the three-stage process, priority was given to the diagnosis reached on the basis of clinical interviews (12). The IQ was estimated on the basis of the vocabulary and block design sub-tests of the Wechsler Intelligence Scale - third edition (WISC - III) (13) administered by a trained psychologist (14).

Although the K-SADS-E is used to reach 
diagnoses according to the DSM-III-R, the version currently in use in our service allows the diagnosis of some categories according to DSM-IV criteria (15). Thus, all patients were assessed for $\mathrm{AD}$ and SP. For the purpose of this study, we relied on diagnoses reached on the basis of the K-SADS-E and reviewed by the clinical committee. In addition, we extensively reviewed all the records of patients with diagnoses of $\mathrm{AD}$ and/or SP. Cases that did not unequivocally meet diagnostic criteria were excluded.

We assessed the following demographic and clinical data of the sample: a) age, b) sex, c) age at onset of symptoms, d) level of impairment, e) number of social situations impaired (only for the groups with SP alone or in combination with $\mathrm{AD}$ ), f) comorbidity with other anxiety disorders, and g) comorbidity with other prevalent mental disorders. To avoid excessive comparisons that would increase the chance of type I error, we first assessed differences among groups in two general categories, one comprising all affective disorders, and the other (disruptive behavior disorders) concerning subjects with attention-deficit/hyperactivity disorder, oppositional defiant disorder or conduct disorder. Only when a difference was detected among groups did we perform specific analyses of each diagnosis that composed the general category.

The Institutional Review Board (IRB) of HCPA (approved as an IRB by the Office for Human Research Protections, United States of America - IRB 00000921) approved the chart review of the sample for the purpose of this study.

We assessed the chance of agreement between $\mathrm{AD}$ and $\mathrm{SP}$ diagnoses in our sample (the chance that $\mathrm{AD}$ is diagnosed in a subject when criteria for SP are also met and viceversa) through the use of the $\kappa$ coefficient. Criteria proposed by Landis and Koch (16) were used to interpret the $\kappa$ coefficients: excellent reliability, $\kappa>0.75$; good reliability, $\kappa=0.59$ to 0.75 ; fair reliability, $\kappa=0.40$ to 0.58 ; poor reliability, $\kappa<0.40$. Comparison of all categorical variables was performed using the chi-square test. Since most of the continuous data did not show normal distribution, the non-parametric Kruskal-Wallis test was used to compare these variables among groups. A 5\% level of significance was accepted in all comparisons (two-tailed).

The sample consisted of 394 children and adolescents assessed in our settings during the study period. We excluded 19 subjects $(4.8 \%)$ from the sample because of a) uncertainty about the diagnoses of $\mathrm{AD}$ or $\mathrm{SP}$ after a review of the records $(\mathrm{N}=16)$, and $\mathrm{b})$ a diagnosis of pervasive development disorder $(\mathrm{N}=3)$. The sample $(\mathrm{N}=375)$ was divided into four groups: a) subjects fulfilling diagnostic criteria for $\mathrm{AD}$ but not for $\mathrm{SP}$ $(\mathrm{N}=7)$, b) subjects fulfilling diagnostic criteria for SP but not for AD $(\mathrm{N}=26), \mathrm{c})$ subjects fulfilling diagnostic criteria for both $\mathrm{AD}$ and $\mathrm{SP}(\mathrm{N}=24)$, and d) subjects that did not fulfill diagnostic criteria for AD or SP (N $=318$ ). The $\kappa$ for the agreement between the two diagnoses was $0.55(\mathrm{P}<0.001)$.

No significant difference was found among groups in age at admission to the study (median age in months for the AD, SP, and $\mathrm{AD}+\mathrm{SP}$ groups was $142.57,135.15$, and 131.29 , respectively; $P=0.74$ ). However, a significant difference in sex was found among groups $(P=0.03)$. Although there was a male preponderance in all three groups, the proportion of males was higher in the $\mathrm{AD}$ group (100\%), intermediate in the SP $+\mathrm{AD}$ group $(83.3 \%)$, and lower in the SP group $(61.5 \%)$.

We detected no significant difference among groups in the pattern of comorbidity either with other anxiety disorders or with different mental disorders (Table 1). However, it is important to note that the number of comorbid conditions in all three groups was high (the median number of comorbidities for the $\mathrm{AD}, \mathrm{SP}$, and $\mathrm{AD}+\mathrm{SP}$ groups was 3,3 , and 4 , respectively; $P=0.58$ ). Even the rate of comorbidity with other anxiety disor- 
ders was high in all groups (the proportion of patients with an additional diagnosis of anxiety disorder for the $\mathrm{AD}, \mathrm{SP}$, and $\mathrm{AD}+\mathrm{SP}$ groups was $57.1,61.5$ and $75 \%$, respectively; $\mathrm{P}=0.51$ ). Similarly, no significant difference was detected in the level of impairment among groups or in the number of impaired social situations between the SP and $\mathrm{SP}+\mathrm{AD}$ groups (Table 1).

Thus, we have demonstrated a clinically significant level of overlapping between AD and SP in a referred sample of children and adolescents. Although only a moderate agreement between the two diagnoses was achieved and a difference in the male/female ratio was detected among groups, none of the clinical variables assessed differed significantly between subjects that presented each condition separately or in combination.

The moderate agreement between the two conditions was not an unexpected finding, since previous studies suggested that several subjects with SP do not fulfill a diagnosis of $\mathrm{AD}$, thus reducing the rate of agreement (4). On the other hand, the great majority of patients with $\mathrm{AD}$ (77.4\%) fulfilled the diagnosis of SP, supporting the view that $\mathrm{AD}$ represents a subgroup inside the SP spectrum. However, it is important to note that

Table 1. Clinical characteristics of the child and adolescent groups studied.

\begin{tabular}{lccc}
\hline Clinical characteristics & $\begin{array}{c}A D \\
(N=7)\end{array}$ & $\begin{array}{c}\text { SP } \\
(N=26)\end{array}$ & $\begin{array}{c}A D+S P \\
(N=24)\end{array}$ \\
\hline $\begin{array}{lccc}\text { Comorbid conditions } \\
\text { Anxiety disorders }\end{array}$ & $4(57.1 \%)$ & $16(61.5 \%)$ & $18(75 \%)$ \\
DBD & $7(100 \%)$ & $21(80.8 \%)$ & $17(70.8 \%)$ \\
Affective disorders & $1(14.3 \%)$ & $11(42.3 \%)$ & $10(41.7 \%)$ \\
Age-of-onset of AD symptoms (months) & $36[24-132]$ & $\mathrm{NA}$ & $66[18-120]$ \\
Age-of-onset of SP symptoms (months) & $\mathrm{NA}$ & $72[24-132]$ & $72[24-108]$ \\
Level of impairment (severe) & $4(66.7 \%)$ & $11(52.4 \%)$ & $8(40 \%)$ \\
Number of social situations impaired & $\mathrm{NA}$ & $1[1-3]$ & $2[1-4]$ \\
& & &
\end{tabular}

$\mathrm{AD}=$ avoidant disorder $\mathrm{SP}=$ social phobia; $\mathrm{DBD}=$ disruptive behavior disorder; $\mathrm{NA}=$ not applicable. Data are reported as medians and range (in brackets) for continuous variables. $\mathrm{N}$ and percentage (in parentheses) are reported for categorical variables. No significant difference was detected for any comparisons among groups (for categorical variables: $\chi^{2}$ test; for continuous variables: Mann-Whitney U-test; $\left.P>0.05\right)$. For level of impairment (severe) data were not available for 1 subject in $A D, 5$ subjects in $\mathrm{SP}$ and 4 subjects in $\mathrm{AD}+\mathrm{SP}$.
$22.6 \%$ of $\mathrm{AD}$ children and adolescents did not fulfill a diagnosis of SP. Probably, this subgroup of patients may have shyness as a behavior trait, but not to such an extent as to determine active phobic symptoms. Whether or not this subgroup of patients will develop other anxiety disorders or personality disorders such as avoidant personality disorder in adulthood is a question that deserves further investigations (17).

The preponderance of males in the three groups is in agreement with some of the few clinical studies found in the literature that assessed SP and AD in children $(2,4)$. Although the exact reason for a significantly higher male/female ratio in the group with $\mathrm{AD}$ diagnosis than in the SP group is unknown, it could be an artifact of the small sample size of the $\mathrm{AD}$ group.

Our findings demonstrating no significant difference in clinical variables among groups agree with those of the only other study in the literature that investigated this question. Francis et al. (8) assessed a sample of 62 children and adolescents with anxiety disorders divided into three groups (AD, SP, $\mathrm{AD}+\mathrm{SP}$ ). They did not detect significant differences among groups regarding most demographic variables or the pattern of comorbidity with affective and other anxiety disorders. Thus, even the subgroup of children with $\mathrm{AD}$ but without SP $(22.6 \%)$ did not present a significantly higher level of impairment, or a significantly different pattern of comorbidity compared to the other groups.

We detected a high rate of comorbidity with other mental disorders, and a very large number of subjects that presented additional anxiety disorders in all groups. Since we were dealing with a referred sample of children and adolescents, these findings were expected. In fact, comorbidity is the rule, and not the exception, in clinical samples of children with mental disorders (18). In addition, the association of one anxiety disorder with comorbidity with other anxiety disor- 
ders is an extremely frequent occurrence in referred samples $(1,4,19)$.

The present findings should be interpreted within the context of some limitations of the present study. The sample size was relatively small, especially for the AD group (N $=7$ ). Thus, we cannot exclude the possibility of a type II error in some comparisons. In addition, we assessed a clinical sample of children and adolescents referred to a highly specialized outpatient facility inside a uni- versity hospital. Thus, the present findings may not be generalized to non-referred samples.

Even considering these caveats, we found more similarities than differences among children with $\mathrm{AD}, \mathrm{SP}$, or both conditions combined. The majority of children with AD fulfilled criteria for SP. Thus, our findings support the validity of the view of AD as part of the SP spectrum in a clinical sample.

\section{References}

1. Bernstein GA \& Borchardt CM (1991). Anxiety disorders of childhood and adolescence: a critical review. Journal of the American Academy of Child and Adolescent Psychiatry, 4: 519-532.

2. Livingston $R$ (1996). Anxiety disorders. In: Lewis M (Editor), Child and Adolescent Psychiatry. A Comprehensive Textbook. 2nd edn. Lippincott Williams \& Wilkins, Baltimore, MD, USA, 674-683.

3. Moutier CY \& Stein MB (1999). The history, epidemiology, and differential diagnosis of social anxiety disorder. Journal of Clinical Psychiatry, 60 (Suppl 9): 4-8.

4. Last CG, Strauss CC \& Francis G (1987). Comorbidity among childhood anxiety disorders. Journal of Nervous and Mental Disease, 12: 726-730.

5. American Psychiatric Association (1994). Diagnostic and Statistical Manual of Mental Diseases (DSM IV. 4th edn. American Psychiatric Association, Washington, DC, USA.

6. American Psychiatric Association (1980). Diagnostic and Statistical Manual of Mental Diseases (DSM III). 3rd edn. American Psychiatric Association, Washington, DC, USA.

7. American Psychiatric Association (1987). Diagnostic and Statistical Manual of Mental Diseases (DSM III-R). 3rd edn. Revised. American Psychiatric Association, Washington, DC, USA.

8. Francis G, Last C \& Strauss CC (1992). Avoidant disorder and social phobia in children and adolescents. Journal of the American Academy of Child and Adolescent Psychiatry, 31: 1086-1089.

9. Otto MW, Pollack MH, Maki KM, Gould RA, Worthington 3rd JJ, Smoller JW \& Rosenbaum JF (2001). Childhood history of anxiety disorders among adults with social phobia: rates, correlates, and comparisons with patients with panic disorder. Depression and Anxiety, 14: 209-213.

10. Orvashel H (1985). Psychiatric interviews suitable for use in re- search with children and adolescents. Psychopharmacology Bulletin, 21: 737-744.

11. Polanzyk G, Eizirik M, Aranovich V, Denardin D, Silva TL, Conceição TV, Pianca TG \& Rohde LA (2003). Interrater reliability for the schedule for affective disorders and schizophrenia. Epidemiological version for school age children (K-SADS-E). Revista Brasileira de Psiquiatria, 25: 87-90.

12. Rohde LA (2002). ADHD in a developing country: Are DSM-IV criteria suitable for culturally different populations? Journal of the American Academy of Child and Adolescent Psychiatry, 41: 1131-1133.

13. Wechsler D (1991). WISC III/Manual. The Psychological Corporation, New York.

14. Sattler J (1998). Psychological Assessment. McGraw-Hill, New York.

15. Kaufman J, Birmaher B, Brent D, Rao U, Flynn C, Moreci P, Williamson D \& Ryan N (1997). Schedule for affective disorders and schizophrenia for school-age children - present and lifetime version (KSADS-PL): initial reliability and validity data. Journal of the American Academy of Child and Adolescent Psychiatry, 36: 980-988.

16. Landis J \& Koch G (1977). The measurement of observer agreement for categorical data. Biometrics, 33: 159-174.

17. Biederman J, Hirshfeld-Becker DR, Rosenbaum JF, Herot C, Friedman D, Snidman N, Kagan J \& Faraone SV (2001). Further evidence of association between behavioral inhibition and social anxiety in children. American Journal of Psychiatry, 158: 1673-1679.

18. Angold A, Costello EJ \& Erkanli A (1999). Comorbidity. Journal of Child Psychology and Psychiatry, 40: 57-87.

19. Last C, Perrin S, Hersen M \& Kazdin A (1992). DSM-III-R anxiety disorders in children: sociodemographic and clinical characteristics. Journal of the American Academy of Child and Adolescent Psychiatry, 31: 1070-1076. 